Volume 8, No.1, January - February 2019

International Journal of Advanced Trends in Computer Science and Engineering

Available Online at http://www.warse.org/IJATCSE/static/pdf/file/ijatcse16812019.pdf

https://doi.org/10.30534/ijatcse/2019/15812019

\title{
Unconstraint 2-Level Cost Optimization Coding for Signal Processing
}

\author{
Anita Kulkarni ${ }^{1}$, Dr. Elizabeth Rani ${ }^{2}$ \\ ${ }^{1}$ Assoc.Prof., EIE Dept.,VVJIET, Bachupally, Hyderabad-India. \\ ${ }^{2}$ Prof.\& Head, EIE Dept., GITAM University, Visakhapatnam, AP-India.
}

\begin{abstract}
Evolution of new technologies has lead to the development of new devices, exchanging data over a diverse network area. Diversity in the propagation model has given diversity in the interference and has made the estimation algorithm limited to certain interference only. However, the increase in the propagation model, the co-channel interferences are increasing rapidly. In the estimation of such interference, recursive filters were proposed with the objective of lower complexity and faster convergence. Recursive B-spline approximation (RBA) is used as an optimal solution in this approach. An time shift kalman filtration (KF) approach to optimize the RBA using weighted least square (WLS) develops the objective of state stabilization in this approach. However, dynamic interference scenario in the propagation model, leads to a large convergence overhead and the bounded cost function constraint the estimation to bounded limits. To develop a unbounded estimation approach, in this paper, a new 2-Level unconstraint estimation approach for Kalman filter stabilization for RBA is proposed. The method defines a open bound to the signal estimation for faster convergence with higher estimation accuracy.
\end{abstract}

Key words: 2-level cost optimization, fast convergence, Coding performance, channel estimation, recursive filter stabilization.

\section{INTRODUCTION}

Interference models are getting diverse in nature. Interference diversity in signal processing has been effective in various estimation approaches, which is dependent on the Interference uncertainty. The retrieval of signal under imperfect conditions is a critical operation in signal processing. In different approach of estimation, feedback estimators are used as a optimal estimation logic, where a feedback error is propagated to get the estimation, the estimation logic work using a Where the Interference mitigation is being carried out and updated to obtain an estimate. In a signal propagation system, the estimation using conventional filter approach find limited with the increase in communication channel effects or the services demand. To achieve the best performance in such system, different methods were suggested in past. Under the channel imperfection condition a frequency selective logic [1] is proposed, where a pilot base estimation approach is developed to estimate the signal under multipath condition. Filter operates for interference tracking in the communication system and encoding demonstration gives a flexible approach. The analysis is a cumulative evaluation of the channel gain and phase in the communication system such as a multi input multi output (MIMO) [2]. It has been put early that feedback estimators follow the extended phase noise. It is observed that the extended filtering approaches are more stable in the estimation of phase tracking noise. A similar approach using the semi-blind approach to estimate the interference is outlined in [3]. It is used for frequent estimation in logical estimation based on feedback using blocks of data bits. Logical estimate was developed based on a dynamic block, taking into account the case of adding the interference quickly and the state of channel diversity in the system is outlined in [4]. To achieve the estimation at faster rate a solution for the interference estimation approach based on the feedback filter is outlined. The state derives transfer coefficient depending on whether the logic of the correct value is limited to a time varying environment. In order to achieve the objective of the user's movement, a new concern in motion based estimation for the $M$ system is proposed in [5, 6]. An approximation method to estimate the interference at different speed of movement in the slow, fast and medium format is observed. It is designed to filter the performance of the feedback channel revealed that the signal contained in the movement case is more interfered compared to static model.

The estimation performance is based on the channel effect observed in the communication process. The efficiency of coding of a interference estimator is concerned with the interference consideration. For the estimation of signal, in [7] an impulse response of channel effect is used, where a correlation logic is developed for a correlative approach for transmit and receive antenna. An approach of integrated LMS filter [8] is used for the optimization of interference estimation in a time frequency domain. The estimation performance under highly varying interference units for time dependent channel effect. For the improvement of estimation performance in multi input system, a symmetric approach was outlined in [9]. The estimation approach is defined to minimize the MSE, power and interference based on DFT approach. The estimation of interference effect using symmetrical estimation model was used for the MSE and power loss minimization. In [10] to estimate the SCM channel for MIMO systems with zero-fill is defined with a distinctive attributes. State verification is very simple and the most relaxed of the column is distributive mode. it can be applied to the case of transmission antennas in particular cases. Through the evaluation of simulation, the error rate performance is observed in the retrieved bits are low to medium in estimation zone. To determine path closer to the best estimation in [11] a second round of the CFO and advances in putting frequency isolation is suggested. Rounding provides first-class replication algorithm to fit to 
estimate the excellent track that the traditional method of the comparison scale. It work in the second round rounding way in improving the frequencies and channels to track estimation as compared to the first form. In [12] channeling long-term estimator attributes is computed through a sub domain mapping algorithm through space-time patterns in the channel. On the other hand, variable fading can be tracked using the techniques used for temporal fade channel coherence. In particular, It was chosen in MIMO based on a BISM equation Turbo reference model for performance evaluation in terms of bit-error rate.

In [13] scheme has been put to coincide with the semi-blind scheme to estimate the time channels for systems and modules based on vectors. In semi-blind coding, the operation was performed through three operative phases, (1) calculating the time difference between the maximum gain in multiple-channel fading, (2) minute sedimentation time algorithm to find a location in the channels, and (3) Reach of the interference response. A methodology is proposed to evaluate the performance of the MIMO systems through optional and overlapping variable frequency channels. In the process of estimation of unknown channel parameter, to mitigate noise interference in signal estimation, recursive filters [14-16] were used. The recursive filters were developed with the objective of minimizing the feedback error by weight optimization $[17,18]$ or by sliding windowing approach [19]. The optimization is developed as a convergence of a cost function defined as a recursive error minimization between the estimate and the actual values. The conventional models developed in past as a constraint of signal estimation by a prior knowledge of the interference, or a complex blind approach is used in estimation. Wherein prior knowledge based has greater accuracy, the maintenance is high, and a blind system has a computation convergence overhead. Towards optimizing the estimation performance in [20], a recursive B-spline approximation (RBA) is proposed. To converge the system faster kalman filter (KF) approach were introduced. Where the estimate derive a interference minimization using a weighted least square (WLS) estimate. The developed system however, doesn't explore the diversity of interference model and the estimation boundness to a interference limits are observed. To develop a faster estimation approach giving the convergence of estimate faster under diverse interference effect is proposed.

To develop the proposed approach, in this paper a new 2level estimation logic applied to a MIMO based signal propagation model is proposed. The proposing approach focus on the minimization of convergence delay, with increase in bit error rate (BER) performance. The rest of this paper is outlined in 6 section, where section 2 outlines the interference models considered in system design. Section 3 outlines the conventional approach of Recursive B-spline approximation and kalman filter optimization. Section 4 outlines the proposed 2-Level unconstraint optimization approach. The simulation result for the developed approach is outlined in section 5. Section 6 presents the conclusion of the developed approach.

\section{SYSTEM MODELING}

In a signal propagation model, the spatial correlation arises because of spacing and weak antenna environments and dispersion in the channel model. This requires a complex approach at the receiver to recover the symbols sent for an accurate estimate of the noise parameter to recover the signal back.

In the time domain, the estimate of the unknown is of the length of the channel. in a communication model, the transmitter transmits the data based on the logical frame count. Depending on the degree of cooperation between the encoding unit and the information bits to transmit, each data spread up to $\mathrm{N}_{\mathrm{I}}$ overlapping or fixed over the capacity of the entire channel length. With the consideration of a coordinated approach, the interference model exchange the original signal as a modulated version of the original data, processed over an interference. This paper examines each of the randomized scheduling policy and the randomness of the communication scenario in the signal propagation scheme. The channel effects the propagating signal with the interference level observed, the estimation is performed based on the recursive modeling or a delay model. In Frequency Selective coding with $\mathrm{N}_{\mathrm{R}} \times N_{\mathrm{T}}$ with Response $H_{k}$, given by,

$H_{k}=G_{k} \sum_{r=1}^{W} \sqrt{P_{r}} A_{r} \exp \left(-j 2 \pi \frac{k}{N}\right)$

Mean power $P$ is the power of each path, $\mathrm{N}_{\mathrm{R}} \times \mathrm{N}_{\mathrm{T}}$ fades capacity. $G_{k}$ is the gain of transmission on the frequency response of the filter, reflecting the fading capacity considered to be consistent with the broad unregulated stable dispersion model given as,

$R_{S, r}=R_{T x, r} \otimes R_{R X, r}$

Fade calculations to match spatial product exhibits a Kronecker product of $R_{T x}$ with $R_{R X}$ spatial cooperation between sending and destinations. If the interference modeling different correlator, the co-matrix is structured and a multi-beam optimization forming a connective strategy to reduce inter-cell interference is derived based on a reference knowledge called pilot is used. Even if the subcarrier-power noise is estimated by changing the actual sub-receiver's average power noise within the data segment. Based on the interference observed the propagation interference is observed to be coordinative or uncoordinative in nature.

\section{a. Coordinated Interference model}

In a coordinated interference model, the signal in the propagation is referred to the neighboring sub neighbors or that the entire data region, $Q_{k}=Q$, for $k \in \mathcal{K}$. Where $k$ is a constant variation on the formation of constant interference patterns and the exact difference of the $\mathrm{Q}$, which can be used efficiently. To increase the estimation at signal overlap, a linear array of uniform adoption (ULA) separated by a distance. To increase the ability to mitigate interference, uniform linear antennas are replaced with varying antennas at each sub-carrier to adopt the SINR beam formation method. $\gamma_{k}=\gamma\left(H_{k} .7\right)$. Assuming the prior interference knowledge, in combination the signals 
contained in different antennas issued the minimum distortion variance in the receiver, by adding the generation feedback output. Changes depending on the interference pattern differs with the signal region in the channel. In this approach of coordinative interference model, the channel is designed with a limiting SINR defined by,

$\gamma_{k}=\frac{p_{o}}{N_{T}} \operatorname{tr}\left\{H_{k}^{H} Q^{-1} H_{k}\right\}$

The interference is observed to be coordinative with the propagation model used in signal propagation. The coordinative interference model is observed under a linear channel model at different sub carrier frequency.

\section{b. Uncoordinated interference model}

In this case the maximum interference is provided by variation and inefficiency aimed at estimating from the estimated value. different estimation approaches in a random access mode that provides overlapping interference is observed at the uncoordinated interference model. In this case, due to the uncertainty of the formation of different frequencies, to reduce interference it is estimated at uneven $Q_{k}$. The maximum receiver reception probability (ML) is based on the detection of the presence of a data at the decoder on the average noise power density of $\sigma^{-2}$. The SINR in this case is given by,

$\gamma_{k}=\frac{p_{o}}{N_{T}} \frac{\operatorname{tr}\left\{H_{k}^{H} Q^{-1} H_{k}\right\}}{\sigma^{2}}$

For minimum error performance, the signal variation at decoder is taken for a SINR with noise power $\sigma k^{2}$ for each Sub carrier as,

$\gamma_{k}=\frac{p_{o}}{N_{T}} \frac{\operatorname{tr}\left\{H_{k}^{H} Q^{-1} H_{k}\right\}}{\sigma k^{2}}$

The channel interference for modeling and evaluation as a measure of channel response given by,

$\hat{h}_{k}=\sqrt{\frac{p_{o}}{N_{T}}} \cdot \operatorname{vec}\left(Q_{k}^{-H / 2} H_{k}\right)$

For the variation $Q_{K}=Q$ at random condition, the interference is taken as $Q_{K}=\sigma^{2} I_{N R}$, the SINR in this case is defined by,

$\gamma_{k}=\left\|\hat{h}_{k}\right\|^{2}$

In this interference model the channel is a diverse and uncertain in estimation. The dynamic interference condition leads to uncertainty and nonlinear characteristic of the channel response. It is hence required to develop estimation under the coordinative and un-coordinative scenarios to obtain estimation performance.

\section{SIGNAL ESTIMATION -LMS RECURSIVE APPROACH}

The imperfection in wireless medium leads to diversity in signal estimation. In the estimation process, the estimator is designed to estimate the data based on estimates or prior information. However, with the increase in demand for greater services and faster data transmission, these estimators are getting limited to processing speed. The concern of such estimator is the convergence time. The convergence issue for an estimator unit is an important requirement in signal estimation. It is required to have a faster convergence to give fast processing efficiency in a wireless communication system. To improve the processing accuracy and overhead minimization in this work, the conventional RBA using KF with WLS estimation [20] is improved. In addition the convergence performance of the recursive estimator is also required to give the system output faster. In the approach, of signal propagation, data are communicated over wireless medium from a source to sink. The propagation model observe different noise effects to the transmitting data resulting in signal degradation. In the propagation mode for a communication system, signal $x_{n}$ is transmitted over a channel, giving the impact of noise effect, the interfered signal is defined by,

$I(t)=\sum_{n=1}^{k} x_{n} \delta(t-N T)$

The signal $x_{n}$ is generated at $1 / \mathrm{T}$ rate processed over a channel response of $h(t)$. A signal delay of $\delta$, giving a time shift of the NT to the original signal time period. The interfered signal $I(t)$ is observed to be an integrated model of all propagated effected signal for paths $1 \ldots \ldots . . k$.

For the estimation the interference from the interfered signal, a feedback estimator logic is used to minimize the interference objective. The convergence performance under the randomized condition is performed using a weighted least square (WLS) approach. The estimation of the signal is performed from the additive noise observed over the transmitted signal $\left(X_{n}\right)$

$I_{n}=X_{n}+N_{n}=\sum_{k} h_{k} x_{n-k}+N_{n}$

Where $x_{n}=x(n T)$

These interferences are to be estimated and equalized to improve the retrieved quality of a receiver unit. Recurrent feedback estimation logic is used as reference error suppression in interference minimization. However, these approaches are accurate when the interference is a dual of the estimator and degrades in quality when the interference is dynamically changing. The estimation, of the degraded signal is carried out using recursive estimators.

In the process of recursive estimation, a recurrent B-spline approximation (RBA) [20] was suggested. This approach derives new estimation function using $b$-spline model where the interference mitigated by a time delay parameter. The error estimation is derived by linear weighted least square method, which computes a recursion of error propagation to derive constant state vector $v$ defined by,

$y_{k}=c_{x_{k}}+n_{k}$

Where $C$ is defined as a correlative matrix of $x$ to $y$. For the estimation of the error and to minimize it, a recursion process is made to obtain stabilization. This stabilization is obtained using a Kalman filter. The estimator tries to estimate the signal, from the received signal stabilizing the state of estimates by a linear estimate equation defined by,

$S_{k}=A x_{k-1}+B u_{k-1}+w_{k-1}$ 
The error estimation is defined as,

$$
E_{k}=H_{x_{k}}+n_{k}
$$

The estimation feedback error is derived from the estimated signal, where $\mathrm{E}$ is taken as the estimated reference derived from the received signal. The measurement of the state stability is governed by the detection of the impulse $\mathrm{H}$ and noise parameter $\mathrm{n}_{\mathrm{k}}$ from the estimate. But it is observed that the a Recursive filter has an assumption which limits its usage under diversity condition. The WLS approach performs the recursion operation to converge the cost function given by,

$$
\dot{X}=\min (y-C x) R^{-1}(y-C x)^{-1}
$$

The defined approach uses the convergence under a linear bounded channel effect. A prior channel knowledge is considered in this case. The convergence is obtained as a minimization of the WLS error computed with the covariance matrix $(R)$. This assumption, leads to open loop issues in estimation and noise suppression, and leads to system instability. Under diverse condition, where the signal travels through multiple paths as defined by $\mathrm{H}$ matrix of $\mathrm{m} \times \mathrm{n}$ size, where ' $\mathrm{m}$ ' is the number of information bit passed from each transmitting antenna with ' $n$ ' simultaneous. This $\mathrm{m} \times \mathrm{n}$ diversity result in imperfect condition, this leads to unstable error. To resolve these issues, a recursive estimator logic using min-max convergence criterion is proposed.

\section{PROPOSED UNCONSTRAINT 2-LEVEL ESTIMATION}

In the proposed approach, the estimation is performed as a recursive estimation, with feedback error. The optimization criterion in this case is kept as a linear range of estimates, rather to a fixed limit as observed in conventional modeling. The least error or minimum square error approach is best applicable to linear model, whereas this estimate is not observed to be optimal in this scenario. To open the estimate limits, a minimum and maximum error bound factor is defined, called as '2-level unconstraint' filter. This open limit leads to an estimation stabilization under diverse condition.

The proposed approach optimizes the open loop estimation error criterion. Here, the proposed unconstraint Filter the filter is defined as a norm of covariance matrix derived over a open bound in a blind manner. The estimate in this case is observed to be uncertain, and estimation of $y$ is predicted. Using the Recursive loop to estimate the state referring estimate of $x$ as $\hat{x}$, the filter equations is given by,

$\hat{x}_{k+1}=\left(A \hat{x}_{k}+B u_{k}\right)+K_{k}\left(y_{k+1} C \hat{x}_{k}\right)$

Where,

$K_{k}=A P_{k} C^{T}\left(C P_{k} C^{T}+S_{z}\right)$

and

$\left.P_{k+1}=A P_{k} A^{T}+S_{w} A P_{k} C^{T} S_{z} C P_{k} A^{T}\right)$
Where $S_{w}$ and $S_{z}$ is covariance matrix of $W$ and $Z$, and $k$ is gain of recursion, and $P$ is given as the variance of the estimation error. The conventional error is observed to be optimal when the average value of the process noise, $W_{k}$, is zero, and the average value of the measurement noise, $Z_{k}$, is zero. However, in a real time scenario under diversity this condition is not achievable, as the time series observation is nonlinear. To overcome this issue, the open bound filter performs a 2-Level estimation defining the unconstraint limits as minimum and maximum estimation limits, where the vector norm of the original signal is computed, defined given by,

$\|x\|=\sqrt{x^{T}} x$

Which is also defined as,

$\|x\|^{2}=x^{T} x$

A weighted vector norm is used to adapt the estimates, and is defined as

$$
\|x\|_{Q}^{2}=x^{T} Q x
$$

Where $Q$ is a diagonal matrix. Taking the weighted norm matrix the system tries to solve the convergence problem of

$\dot{X}=x^{T} x \min _{\widehat{x}} \max _{w, v} M$

Where $M$ is the cost function defining the estimation limit computing the noise terms $W$ and $v$ which are dynamic in nature. The cost function $M$ is defined as a ratio of the estimate of average correlative value over the average estimates $w$ and $v$. The cost function hence converge w.r.t. the estimated noise over the correlation of the signal, building a equilibrium of correlation over the estimate. The cost function is defined by,

$M=\frac{a v e\left\|x_{k} \hat{x}_{k}\right\|_{Q}}{\text { ave }\left\|w_{k}\right\|_{w}+a v e\left\|v_{k}\right\|_{v}}$

Where the averages are taken over all time samples $k$. Here the estimate tries to minimize the cost function $M$, to find a $\hat{x}$ that is close to the original signal $x$. The average weighted norm function in the denominator of the cost function, relate to the minimizing of the cost function, wherein, the improvement in the estimation of original signal $x$ is observed at the numerator term, relating to the maximization of estimation accuracy. This builds to the equilibrium criterion of min-max stabilization. The estimate where the two terms result in $\min M$ is then considered as the estimate value for equalization.

\section{SIMULATION RESULTS}

The proposed estimation coding for successive and randomized estimates is evaluated for different noise density. The frequency response of such filter is observed in Figure 1. 


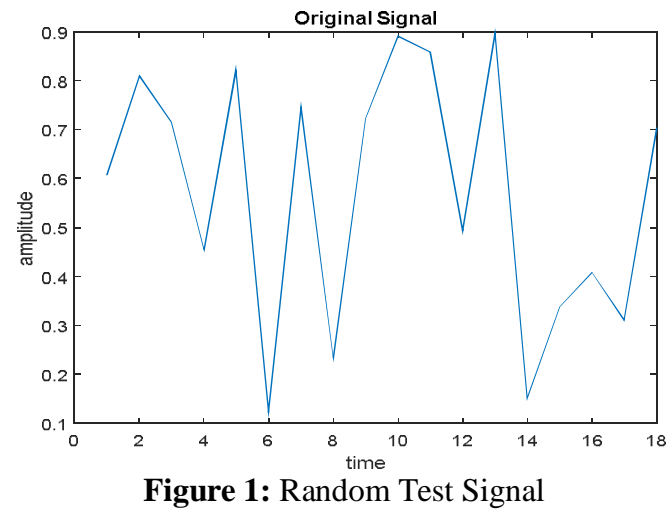

A test sample in a random format is generated; the input sample is shown in figure 1. This input signal is modulated using frequency modulation and processed with additive Gaussian noise, and adaptive filtrations were applied to estimate the signal. The measuring parameter of Mean square deviation (MSD) and convergence time is computed. The developed approach is evaluated over different values of parameters. The original test signal is propagated via 4 interference model, with each frequency resolution isolate. To evaluate the estimation efficiency of the two interference model (coordinated and random), Normalized MSD is computed. The obtained MSD for the two methods is as outlined in figure 2.

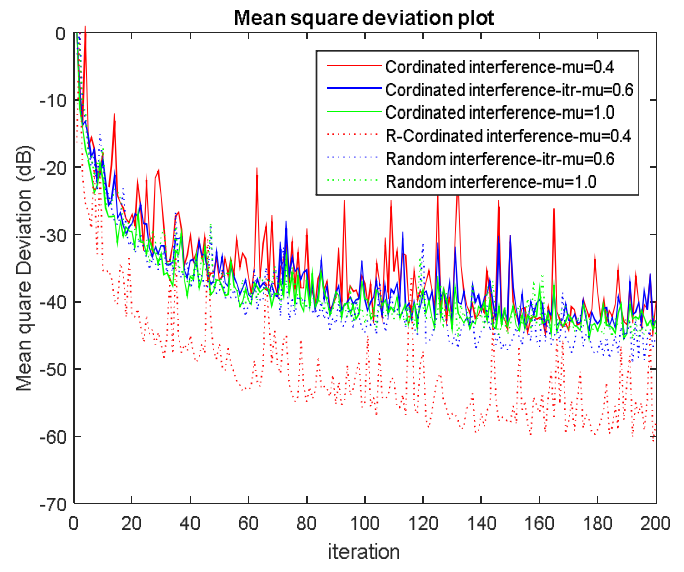

Figure 2: Normalized MSD for $\mathrm{N}=4$, at $\sigma=0.8$

The normalized MSD for the methods developed is presented in figure 2. The noise variance is fixed to 0.8 and the estimation iteration is carried out for 200, at a updating step size of 1.0. The obtained observation illustrates that the Normalized MSD are almost in equality, and a minor decrement of MSD is observed. As the number of signal processing is 4 , the probability of selecting optimal compensation is almost equal in both the cases, leading to very near values.

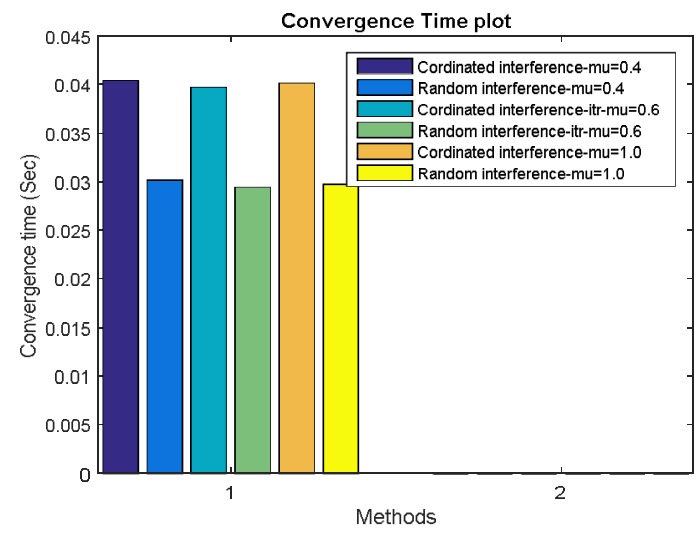

Figure 3: Convergence time measured for the two methods

The process of estimation, however takes lower convergence time due to faster error minimization, as the estimation is distributed over a range of Frequency. Though the Proposed estimation approach iterates over all Kiterations, the convergence time taken for the proposed method is less, due to the fact that the iteration terminates once the limiting factor is achieved as shown in figure 3 . In the case of Randomized approach as all Frequency are processed, a more probability of optimal selection is achieved than the uncoordinated approach. The deviation of estimation under different channel variance is illustrated in figure 4 and 5 .

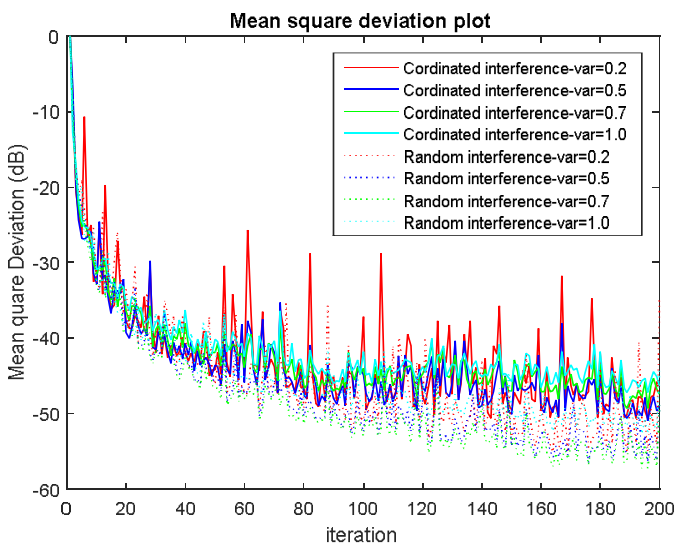

Figure 4: MSD Plot for the interference model at different variance 


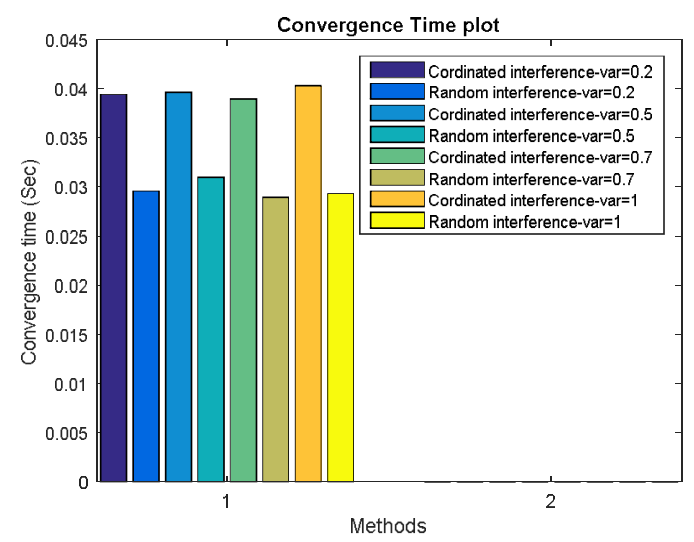

Figure 5: Convergence plot for the developed approach at different variance

The proposed approach is evaluated for a random propagated signal in MIMO model under randomized condition. The developed approach of open bound estimation modeling is used for estimation and compared with the conventional WLS estimation approach. A random set of information bits is taken as input bits. A set of the processing bit is shown in Figure 6.

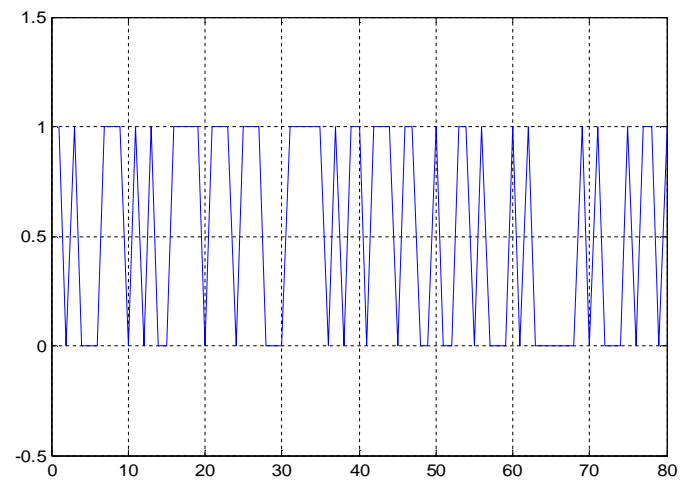

Figure. 6: Original message signal

These information bits are modulated using a Quadrature modulation scheme, where the real distribution of the complex modulated data is shown in Figure 7.

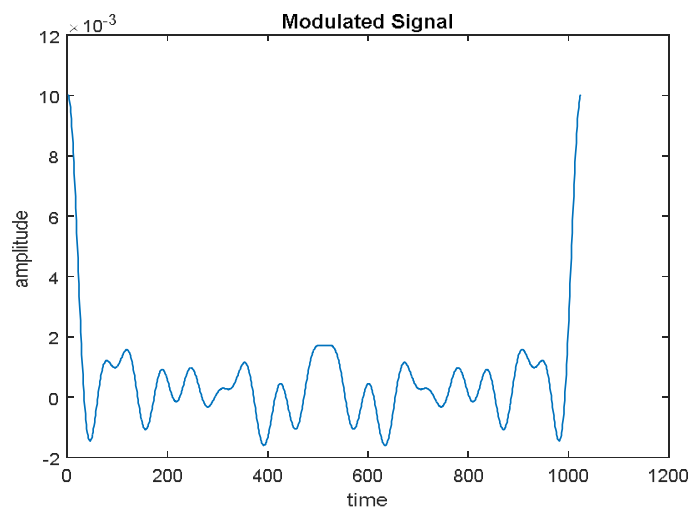

Figure 7: Modulated signal output
This information bit is block coded and transmitted over the time. The effected signal for this transmitted bit is shown in Figure 8 .

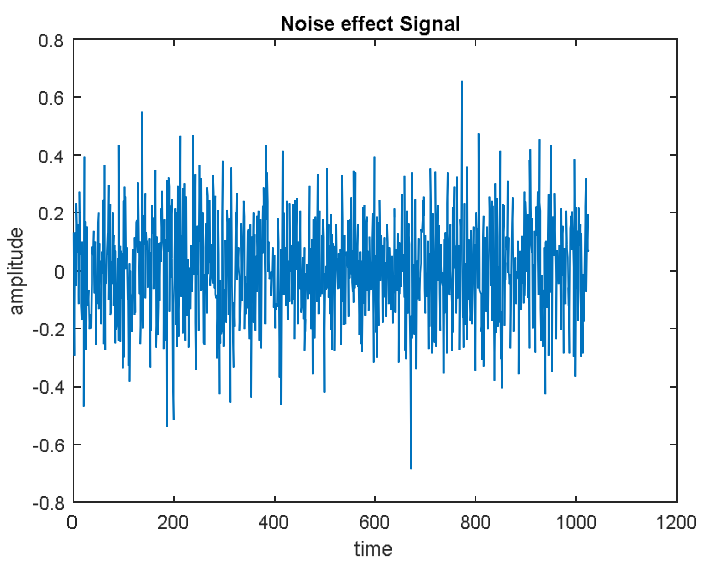

Figure 8: Noise effected signal

The error performance of the developed approach for different time delay is illustrated in Figure 9.

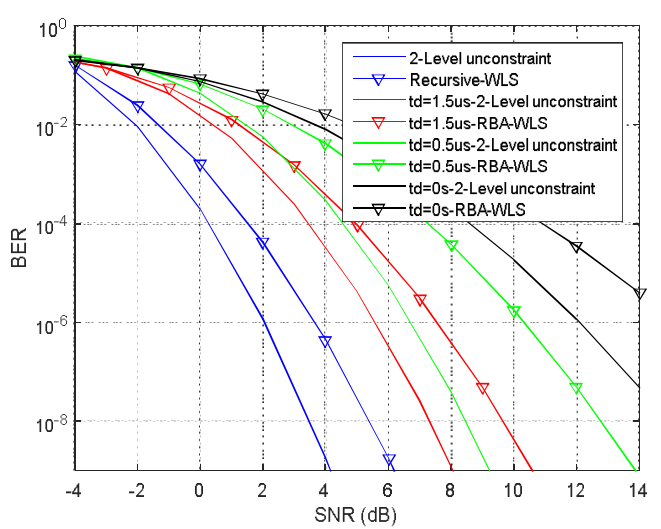

Figure 9: SNR V/s BER comparison plot for 2-Level unconstraint with conventional approaches

The above graph gives the observation for SNR v/s BER of both the conventional and proposed methods. It is clearly observed that the bit error rate of conventional approaches is more when compared with the proposed 2-Level unconstraint approach. A similar test is carried out for a simulation parameter of noise density of $14 \mathrm{~dB}$, and a fading factor of $15 \mathrm{KHz}$, is simulated. The observed result is shown in Figure 10 below. 


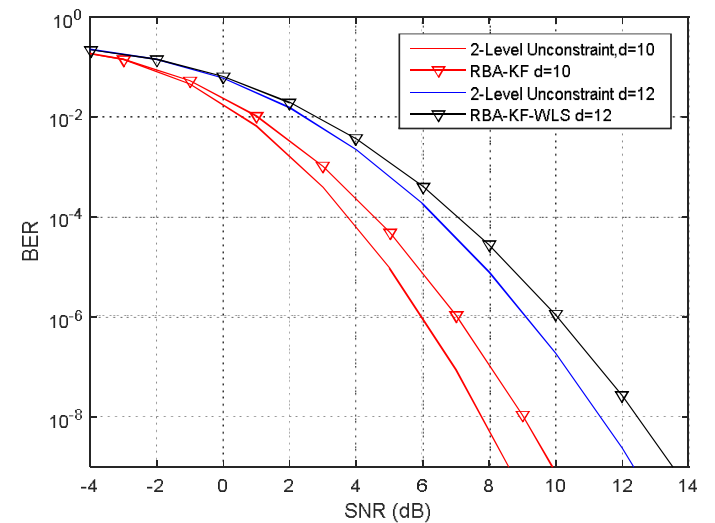

Figure 10: Simulation result of the developed approach under noise density of $14 \mathrm{~dB}$ and fading factor of $15 \mathrm{KHz}$

\section{CONCLUSION}

A unconstraint 2-Level convergence logic is developed for a MIMO propagation model. The approach defines a new cost optimization for MIMO propagation systems, where the is diverse in condition. The randomized condition as observed in real time scenario is simulated for the developed approach, and evaluated under different condition. The observation obtained defines the performance improvement of the suggested approach, where the convergence of the proposed system is observed to be achieved at $0.25 \mathrm{Sec}$ as in comparison to 0.28 by RBS-KF-WLS system. The error performance is also improved due to faster estimation process, which is reduced to be 0.0038 for the proposed model as compared to 0.0012 for the conventional RBS-KF-WLS approach.

\section{REFERENCES}

[1] MingFei Siyau, Tiancheng Li, Javier Prieto, Juan Corchado, Javier Bajo, "A Novel Pilot Expansion Approach for MIMO Estimation and Tracking”, IEEE,ICUWB, pp.1-5, 2015.

[2] Mingyang Chen, Wenwu Wang, Mark Barnard and Jonathon Chambers, "Wideband DoA Estimation Based on Joint Optimisation of Array and Spatial Sparsity", $25^{\text {th }}$ European Signal Processing Conference (EUSIPCO), 2017. https://doi.org/10.23919/EUSIPCO.2017.8081581

[3] Hemant Gavaskar and Sandeep Kumar Agrawal, " Semi Blind Channel Estimation with Training-Based Pilot in AF Two-Way Relaying Networks", International Journal of Current Engineering and Technology, Vol.7, No.1, 2017.

[4] Shijie Zhang, Dan Wang and Jun Zhao "A Kalman Filtering Estimation Method Based on State Transfer Coefficient Using Threshold Correction for UWB Systems", International Journal of Future Generation Communication and Networking, Vol.7, No.1, pp.117-124, 2014.

https://doi.org/10.14257/ijfgcn.2014.7.1.12
[5] Saeed Sepasi, Leon R. Roose and Marc M. Matsuura, "Extended Kalman Filter with a Fuzzy Method for Accurate Battery Pack State of Charge Estimation”, Energies, vol.8, pp-5217-5233, 2015.

[6] Yunlv Hong, Di He, "An Improved Doppler Frequency Offset Estimation Algorithm of OFDM System under High-speed Movement Environment", Journal of Computers, Vol. 8, No. 12, 2013.

[7] Chin-Te Chiang and Carrson C. Fung, "Robust Training Sequence Design for Spatially Correlated MIMO Channel Estimation", IEEE Transactions on Vehicular Technology, Vol. 60, No. 7, 2011. https://doi.org/10.1109/TVT.2011.2161640

[8] Thiernithi Variddhisaï , Danilo P. Mandic, “On an RLS-like LMS adaptive filter", IEEE, $22^{\text {nd }}$ International Conference on Digital Signal Processing (DSP), 2017. https://doi.org/10.1109/ICDSP.2017.8096130

[9] Józef Borkowski, Dariusz Kania, "Interpolated-DFTBased Fast and Accurate Amplitude and Phase Estimation for the Control of Power", Metrology Measurement System, Vol. 23, No. 1, pp. 13-26, 2016. https://doi.org/10.1515/mms-2016-0013

[10] Iyad Hashlamon, Kemalettin Erbatur, "An improved real-time adaptive Kalman filter with recursive noise covariance updating rules", Turkish Journal of Electrical Engineering \& Computer Science, vol.24, pp524-540, 2016.

[11] Roee Shaked, Nir Shlezinger, and Ron Dabora, “Joint Carrier Frequency Offset and Channel Impulse Response Estimation for Linear Periodic Channels", $25^{\text {th }}$ European Signal Processing Conference (EUSIPCO), 2017.

https://doi.org/10.23919/EUSIPCO.2017.8081331

[12] Yunsick Sung, "RSSI-Based Distance Estimation Framework Using a Kalman Filter for Sustainable Indoor Computing Environments", Journal Sustainability, Vol.8, No.11, 2016. https://doi.org/10.3390/su8111136

[13] Te-Lung Kung and Keshab K Parhi, "Semi blind frequency-domain timing Synchronization and estimation for OFDM systems", EURASIP Journal on Advances in Signal Processing, Springer, 2013.

[14] C. Deng, H. Lin, "Progressive and iterative approximation for least squares Bspline curve and surface fitting", Computer Aided Design, Vol. 47, pp32-44, 2014.

[15] Trusila Monyenye Nyandika, George Okeyo, Michael Kimwele, "Enhancing Service Availability during Handover in Wireless Communication-Based Train Control Systems", International Journal of Advanced Trends in Computer Science and Engineering, Vol.7, No.3, pp-41- 51, 2018.

[16] Steven Van Vaerenbergh, Javier V1a and Ignacio Santamar1a, "A Sliding-Window kernel RLS Algorithm and its application to Nonlinear Channel identification", ICASSP, IEEE, 2006.

[17] Hui Zhao, Juhao Li, Paikun Zhu, Cheng Zhang, Yue Liu, Yuping Zhao, Yongqi He, and Zhangyuan Chen, "Weighted Inter-frame Averaging-Based Estimation 
for CO-OFDM System", IEEE Photonics Journal, Vol.5, No.6, 2013. https://doi.org/10.1109/JPHOT.2013.2292355

[18] W. Wang, J. Zhao, "Inverse updating and down dating for weighted linear least squares using minvariant reflections", Linear Algebra and its Application, Vol. 291 No.1, pp-185-199, 1999.

[19] Jesse Hoagg, Asad A. Ali, Magnus Mossberg, and Dennis S. Bernstein, B., "Sliding Window Recursive
Quadratic Optimization with Variable Regularization", American Control Conference, 2011. https://doi.org/10.1109/ACC.2011.5991159

[20] Jens Jauch, Felix Bleimund, Stephan Rhode, Frank Gauterin, "Recursive B-spline approximation using the Kalman filter", Engineering Science and Technology, an International Journal, Vol.20, pp-28-34, 2017. 